\title{
HPC - Health Professional Card oder Highly Problematic Card
}

Heinz Bhend, Andreas Meer, Marco Zoller, Urs Dürrenmatt

\begin{abstract}
Ausgangslage*
Die Notwendigkeit für eine eindeutige Identifikation eines Leistungserbringers wurde vom BAG im Hinblick auf die Erfassung von Gesundheitsdaten auf der Versichertenkarte gefordert. Obschon diese Absicht zwischenzeitlich überholt ist, wird die ein-eindeutige digitale Identifikation der Leistungserbringer mittelfristig notwendig und nützlich sein. Es stimmt nachdenklich, dass ohne Vorliegen einer konsensfähigen E-HealthStrategie der FMH bereits ein Auftrag mit detaillierten Anforderungen dazu vergeben wurde. Dies erstaunt umso mehr, als dass in vielen Praxen bereits heute eine Public-Privat-Key-Sicherheitsinfrastruktur vorhanden ist (HIN), die den Grossteil künftiger Anforderungen abdecken kann. Welche relevanten Praxisprozesse dadurch nicht abgedeckt werden können und den Mehraufwand einer HPC rechtfertigen, wurde bisher nicht kommuniziert.
\end{abstract}

\section{Die Problematik der HPC-Lancierung}

\section{Do not use a computer to force a change of behaviour}

Die Formulierung strategischer Ziele bedingt eine zugrundeliegende Strategie. Die FMH hat bis heute noch keine ausformulierte E-HealthStrategie. Trotzdem wird die HPC seitens unserer Standesvertretung als erstes E-Health-Ziel avisiert. Mit einem solchen Vorgehen läuft man Gefahr, sich an den technischen Möglichkeiten anstelle der tatsächlichen Bedürfnisse zu orientieren. Bevor ein neues IT-Produkt eingeführt wird, sollten die involvierten Strukturen und Prozesse analysiert und gegebenenfalls angepasst werden. Der unlängst kommunizierte Grundsatz der FMH, dass E-Health-Projekte ohne Einbezug der Ärzteschaft nicht erfolgreich sein können, gilt auch für die HPC [1].

\section{Elektronische Dokumentation zuerst}

E-Health aus der Sicht unseres Praxisalltages bedeutet in erster Linie elektronische Dokumentation und Kommunikation. Die HPC zielt auf die technische Regelung der elektronischen Kommunikation ab. Dies, obwohl in der Schweiz erst zirka 12\% der Kollegen eine elektronische Kran- kengeschichte führen. Dieser markante Rückstand im Vergleich zum europäischen Ausland wird durch die HPC nicht wettgemacht. Vor der elektronischen Kommunikation muss die digitale Dokumentation stehen. Es werden weit höhere Geldbeträge notwendig sein, um die Basis auf dem Weg «going paperless» zu begleiten und diesen Umstellungsprozess praxisverträglich $\mathrm{zu}$ gestalten.

\section{Wir pflegen den digitalen Patienten - und werden dafür honoriert}

Es wird argumentiert, dass durch die zügige Einführung der HPC «das Feld besetzt» werden soll. Hinter dieser Argumentation steht die Befürchtung, dass die Ärzteschaft die Kontrolle über diesen wichtigen Identifizierungsprozess verlieren könnte. Das ist möglich.

Dem steht gegenüber, dass unsere Vorleistungen später zum Nulltarif abgeholt werden könnten, da wir die Infrastruktur dann ja schon haben werden und wohl kaum im Nachhinein Finanzierungen erwarten können. Mit der durchaus sinnvollen zunehmenden Digitalisierung der Arztpraxen wachsen die Begehren, die Daten zu Beginn des Patientenpfades korrekt und transferierbar zu erfassen. Da werden enorme Anstrengungen (= Investitionen) nötig sein, um die elektronische Dokumentation so zu implementieren, dass die Informationen mit vertretbarem Aufwand weiter verwendet d.h. transferiert werden können. Die Pflege des digitalen Patienten eröffnet uns Ärzten allenfalls neue «Geschäftsfelder». Bei optimalen Bedingungen leistet die inskünftig ins medienbruchfreie E-Health-Netz eingebundene Arztpraxis Vorleistungen für das ganze System. Wenn wir hier Vorinvestitionen leisten, sollte über mögliche neue Dienstleistungen, Produkte und einen adäquaten Mittelrückfluss nachgedacht werden.

\section{Fehlende Rahmenbedingungen - Datenschutz}

BAG, FMH, E-Health-Strategen gehen von einer Arztpraxis aus, welche selbstverständlich und rund um die Uhr ans Internet angeschlossen ist. Dagegen schreibt der eidgenössische Datenschützer, dass möglichst nur eine separate Arbeitssta- 
tion mit dem Internet verbunden sein sollte [2]. Diese Vorgabe ist unrealistisch, bisher fehlen aber verbindliche Richtlinien des FMH-Rechtsdienstes, unter welchen Bedingungen ein Praxiscomputer mit sensiblen Patientendaten dauernd am Internet sein darf. Ohne diese Voraussetzung ist aber eine rationelle und sinnvolle Anwendung der HPC nicht denkbar. Welche Schutzmassnahmen zusätzlich vorgeschrieben oder empfohlen werden, steht noch nirgends in verbindlicher Form. Sobald diese vorliegt, können entsprechende Folgekosten abgeschätzt werden.

\section{Mögliche Anwendungen der HPC}

Bisher wurde wenig über praxisrelevante, konkrete Anwendungsmöglichkeiten der HPC mitgeteilt. Folgende Geschäftsfälle wurden seitens der Arbeitsgruppe bis anhin identifiziert:

\section{E-Rezept}

Eine konkrete mögliche Anwendung der HPC ergibt sich bei der elektronischen Verschreibung. Hier wird Optimierungs- und Sparpotential erwartet. Dieser Prozess funktioniert indes heute schon digital (z.B. via HIN-Account). Die elektronische Rezeptierung an eine Karte zu binden, läuft Gefahr, den Ablauf in der Praxis unnötig zu komplizieren. Auch bei dieser konkreten Anwendung müsste vor den Vorinvestitionen geklärt werden, ob beispielsweise die Administrativpauschale der Apotheke der elektronisch verschreibenden Praxis zugute kommen könnte. Ferner wäre auch die Übermittlung, Authentifizierung und Validierung von Betäubungsmittelrezepten zu ermöglichen. Hier könnte ein in der Praxis spürbarer Mehrwert erzielt werden.

\section{Berichte senden / Spitalberichte abholen}

Analog zum E-Rezept sollte das digitale Berichtswesen möglichst einfach und praktikabel sein und darf folglich nicht an eine Karte gebunden werden. Die zum Einsatz kommende Authentifizierungstechnologie sollte es ermöglichen, dass Berichte in delegierter Verantwortung durch die MPA transferiert werden können, ohne dass die physische Präsenz der HPC notwendig ist (z. B. hardwarebasierte Authentifizierung). Ferner ist darauf zu achten, dass nicht vom heutigen Bringprinzip zum Holprinzip gewechselt wird. Dies würde einen deutlichen Mehraufwand in der Praxis bedeuten.

\section{Ausblick / Fazit}

Alea iacta est - Der Würfel ist geworfen; aber eben noch nicht gefallen, d.h. das Ergebnis ist noch unklar! Die HPC wird kommen. Die E-HealthEvangelisierung der Ärzteschaft wird noch Jahre dauern.

Ohne eine kritische Masse von Kollegen, die elektronisch dokumentieren, wird die HPC zum teuren Nischenprodukt. Organisationsentwicklung durch Technologie et vice versa braucht ihre Zeit und Geduld. Den Lernprozess dazu haben wir eben erst begonnen.

Bis zum breitflächigen Bedarf einer HPC wird möglicherweise der Fingerscanner, begleitet von einer hardwarebasierten Autorisierungs- und Zertifizierungsroutine, «state of the art» sein.

Vorgängig ist noch genau festzulegen, welche Prozesse zwingend an unseren Finger gebunden werden müssen und welche wir an das Praxisteam delegieren können.

\section{Anmerkungen}

1 FMH-Informationsveranstaltung E-Health/HPC vom 3. September 2008.

2 Eidgenössischer Datenschutzbeauftragter. Leitfaden für die Bearbeitung von Personendaten im medizinischen Bereich - Ausgabe Juli 2002; S. 31. 